\title{
High-performance mixed-matrix membranes with altered interfacial and surface chemistry through benign reinforcement of functionalized carbon nanotubes of different configurations
}

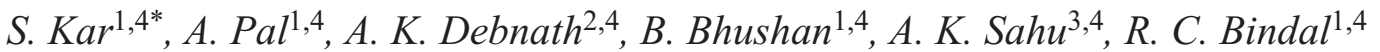 \\ ${ }^{1}$ Membrane Development Section, Chemical Engineering Group, Bhabha Atomic Research Centre, Trombay, 400085 \\ Mumbai, India \\ ${ }^{2}$ Technical Physics Division, Bhabha Atomic Research Centre, Trombay, 400085 Mumbai, India \\ ${ }^{3}$ Glass \& Advanced Materials Division, Bhabha Atomic Research Centre, Trombay, 400085 Mumbai, India \\ ${ }^{4}$ Homi Bhabha National Institute, 400094 Mumbai, India
}

Received 18 December 2016; accepted in revised form 12 February 2017

\begin{abstract}
Nanomaterials potentially minimize the inherent trade-off between productivity and selectivity of membranebased ultrafiltration (UF) process. A comparative study on the reinforcement effect of pristine carbon nanotubes (CNTs) of three different configurations, viz. single-walled (SWNT), double-walled (DWNT) and multi-walled (MWNT), and their carboxylated counterparts, onto a polysulfone (Psf) host matrix of mixed-matrix UF membranes is illustrated herein. The varying structural features of carboxylated CNTs, probed by XPS analysis, underpin the enrichment of CNTs with oxygen rich functionalities following the trend of MWNT $>$ DWNT $>$ SWNT. The membranes with enhanced hydrophilicity and altered electrokinetics substantiate the efficacy of facilitated reinforcement of functionalized CNTs over the pristine ones. Variations in surface topography and mechanical feature of the membranes elucidate that carboxylation influences the interfacial chemistry by enhancing the dispersion stability of MWNTs more profoundly than its configurational counterparts like SWNTs and DWNTs, and concurrently alters its distribution within the membranous matrix. The enhanced ultrafiltration performances, as achieved by twofold enhancement in solvent fluxes without compromise in the solute rejection capabilities ( $\sim 89-90 \%$ toward PEG, $M_{\mathrm{w}}: 35 \mathrm{kDa}$ ), confirm the potential of carboxylated CNTs in leading to development of high-performance mixed-matrix membranes.
\end{abstract}

Keywords: polymer membranes, carboxylation, carbon nanotubes, mixed-matrix, ultrafiltration

\section{Introduction}

Nanotechnology, with tremendous versatility and unprecedented opportunities, has ubiquitously gained the recognition in enhancing the sustainability of membrane-based water treatment processes $[1,2]$. The promising synergistic effects of nano- and membrane technologies provide tangible ways to develop mixed-matrices with tunable functional features, which can make the membrane separation process more productive, energy-efficient, and environmental-friendly. Amongst the nanomaterials, the carbon nanotubes (CNTs) possess outstanding structural, mechanical and electronic properties that are exploited to develop high-performance mixed-matrix systems [3]. The importance of CNTs to the membrane scientists stems from its unique potential to result in a membrane with improved permeability $[4,5]$, gatekeeper controlled selectivity [6], and antifouling 
properties [7-9]. The significance of an integrated composite system of CNTs, with high selectivity for the chemicals of interest as well as a large molecular flux with minimized driving force, has also been emphasized by researchers [10]. In a study carried out by Yin et al. [11], it was demonstrated that oxidized MWNTs at varying concentrations, ranging from 0 to $1 \mathrm{wt} \%$ enhance the Psf based mixed-matrix hollow fiber membranes pure water flux from 60 to $100 \%$ with similar solute rejection abilities. Park et al. [12] investigated the inevitable issues like poor miscibility between the polymeric phase and the CNTs and the resulting phase separation problems for the mixedmatrix membranes. With the aid of experiments and multiscale modeling approaches, the study highlighted the necessicity of functionalization of MWNTs with polar groups like $-\mathrm{OH}$ and $-\mathrm{NH}_{2}$ in order to obtain a benign homogeneous dispersion of the nanofiller within a co-polyimide (P84) matrix to mitigate the phase separation problems. In another study on the CNT reinforced Psf UF membranes, the extent of carboxylation of MWNTs was correlated with the beneficial effect of membranes increased tensile strength, surface hydrophilicity, and permeability as well as the concurrent drawbacks of decreased retention, increased leaching, and decreased strength of CNTs with greater carboxylation [13].

The CNTs are composed of concentric arrangement of several cylinders [14]. As seamless cylinders of single layer of graphene, SWNTs acquire unique properties which can significantly change with the chiral vector [15]. The MWNTs consist of multiple rolled layers of graphene, but still have not been well-defined due to their structural complexity and variety as compared to the SWNTs. The physicochemical properties of SWNTs can change on functionalization, which is attributable to the structural defects occurred by $\mathrm{C}=\mathrm{C}$ bond breakages during chemical processes [15]. However, intrinsic properties of MWNTs can be preserved by surface modification, since the outer walls of MWNTs get preferentially exposed during chemical modification. Lying between SWNTs and MWNTs, DWNTs comprise of exactly two concentric nanotubes that exert properties intermediate between the two other classes. Selective functionalization of the outer walls has led to the use of DWNTs as core-shell systems made of a pristine CNT core and chemically-functionalized nanotube shells [16]. The scope and ease of functionalization of the CNTs offer productive avenues to translate the potential benefits of CNTs onto a membrane matrix. There are several approaches to tune the surface chemistry of CNTs, such as the defect functionalization through covalent attachment of organic moieties to the oxygenated functionality generated at the defect sites of CNTs, and the non-covalent functionalization through secondary $\pi-\pi$ interactions between the outer walls of CNTs and the polynuclear aromatic compounds or conjugated polymers, without affecting the conjugated structures of CNTs. However, the covalent functionalization of $\pi$-conjugated graphitic skeleton by different chemical entities is considered to be more striking as that can exert a superior flexibility in molecular design of the CNTs [17-19]. The most important implication of introducing suitable organic entities is demonstrated by the fact that the attractive van der Waals forces existing between the individual CNTs get reduced, and thereby enhance the thermodynamic stability and the dispersibility of CNTs in aqueous medium or suitable organic solvents of different polarity [20, 21]. The covalent and non-covalent attachment of organic moieties at the sidewalls, end-tips or defect sites of CNTs thus provide better physicochemical compatibility to the nanotubes in host matrices, and thus yield benign reinforcement in mixed-matrices [22, 23].

Although the unique attributes of CNTs that can be translated onto membrane matrix are well-known, this study reported herein holds a comparative discussion on the reinforcement effect of CNTs of different configurations, viz. SWNT, DWNT and MWNT along with their functionalized counterparts, in Psf membrane matrix. Our study looks into the intrinsic relations between physicochemical variations of the CNT reinforced mixed-matrix UF membranes and their overall performance. The effects of functionalized CNTs with varying configurations on the surface topography as well as mechanical features of the mixed-matrix UF membranes have been investigated. The study also evaluates the effect of altered surface chemistry on the performance of the UF membranes through cross-flow ultrafiltration experiments.

\section{Experimental}

\subsection{Materials}

The base polymer, polysulfone (Psf; $M_{\mathrm{w}}: 30 \mathrm{kDa}$ ) was obtained from Solvay Specialities India Pvt. Ltd, India. The solvent, N-methyl-2-pyrrolidone (NMP) with minimum assay of $99.5 \%$ and porogen, polyvinyl pyrrolidone (PVP, K-30; $M_{\mathrm{w}}$ : $40 \mathrm{kDa}$ ) of AR 
grade, as utilized without further purification were procured from SRL Pvt. Ltd. (India). The nanoparticles: single-walled carbon nanotube (SWNT, diameter: $0.7-1.3 \mathrm{~nm}$ ); double-walled carbon nanotube (DWNT, diameter: $5 \mathrm{~nm}$ ) and multi-walled carbon nanotube (MWNT, diameter: 6-13 nm) were procured from Aldrich. For evaluation of membranes' rejection behaviours toward organic solutes, poly(ethylene glycol) (PEG, $M_{\mathrm{w}}: 35 \mathrm{kDa}$ ) and poly(ethylene oxide) (PEO, $M_{\mathrm{w}}: 100 \mathrm{kDa}$ ) were procured from Sigma-Aldrich. The conductivity of Mili-Q ultra-pure water used in the experiments was below $2 \mu \mathrm{S} / \mathrm{cm}$.

\subsection{Synthetic route adopted for surface modification of CNTs}

The oxidative surface modification in solution-state was accomplished using $500 \mathrm{mg}$ of each pristine CNT, i.e., SWNT, DWNT and MWNT. The solutions made in $100 \mathrm{~mL}$ of acid mixture comprising of $1: 1$ (v/v) concentrated $\mathrm{HNO}_{3}(65 \%)$ and $\mathrm{HCl}(37 \%)$, were initially ultrasonicated at ambient condition for $30 \mathrm{~min}$, using a water bath sonicator (Life-Care Equipments Pvt. Ltd., India, $40 \pm 0.3 \mathrm{kHz}, 100 \mathrm{~W})$. After the ultrasonic agitation induced shear stress, the absence of CNT aggregates in all the dispersions was confirmed by visual inspection. Then the sonicated dispersions were refluxed under magnetic stirring at $80 \pm 1^{\circ} \mathrm{C}$ for $4 \mathrm{~h}$, without any further exposure to ultrasonication. Such vigorous oxidative exposure removed the catalyst particles, and concurrently introduced oxygen rich functionalities on the exploited CNTs. The resulting suspensions of functionalized CNTs were allowed to cool at room temperature, followed by filtration through $0.20 \mu \mathrm{m}$ Polypropylene (PP) membrane. The retained solid products were washed several times with water to exclude the residual acids until $\mathrm{pH}$ of filtrates reached $7.0 \pm 0.3$, and then dried in a vacuum oven at $100^{\circ} \mathrm{C}$ for overnight. The carboxylated SWNT, DWNT and MWNT were denoted as C-SWNT, C-DWNT and C-MWNT, respectively, for the ease of representations.

\subsection{Preparation of mixed-matrix UF membranes by non-solvent induced phase inversion method}

For fabrication of mixed-matrix UF membranes in sheet configurations, polymer dope solutions, each having two different compositions were prepared by employing varying proportions of SWNT, DWNT and MWNT as well as their functionalized counterparts:
C-SWNT, C-DWNT and C-MWNT, in hermetically sealed glass bottles. More specifically, the amount of the nanoadditive was varied as 0.25 and $1\left(\mathrm{w} / \mathrm{w}_{\mathrm{Psf}}\right) \%$ in the dope solutions comprising of $20\left(\mathrm{w} / \mathrm{v}_{\mathrm{NMP}}\right) \%$ of Psf and $40\left(\mathrm{w} / \mathrm{w}_{\mathrm{Psf}}\right) \%$ of porogen, PVP. The nanoparticles were dispersed in organic amide solvent, NMP by ultrasonic treatment for $30 \mathrm{~min}$, prior to addition and subsequent incorporation of dried Psf beads and PVP, maintaining the specified compositions. All the dope solutions were then vigorously agitated in mechanical shakers for several hours to achieve complete dissolution of Psf and PVP in the solvent, with homogeneously dispersed CNTs. For comparison purpose, one dope solution (Control), devoid of any nanoadditive was further prepared following the aforementioned composition of Psf and PVP, as well as the methodology. Then, the resultant viscous dope solutions were kept for overnight in an environmentally controlled atmosphere maintaining the temperature and relative humidity at $25 \pm 1{ }^{\circ} \mathrm{C}$ and $35-40 \%$, respectively, to eliminate the trapped air bubbles from the solutions.

To fabricate mixed-matrix UF membranes along with the control one required for comparison purpose, clean and smooth glass plates (without having any fabric-base) were firstly taped at their parallel ends in such a way that each resulting membrane layer could achieve an estimated thickness of $200 \mu \mathrm{m}$. The as-prepared stable dope solutions and the Control were manually cast onto the taped glass plates at a steady casting shear with the help of a well-dried, ultra-smooth glass-roller. The entire assemblies with the cast thin films were immediately immersed in a precipitation bath containing ultra-pure water as the non-solvent, maintained at room temperature, for immersion precipitation. To ensure the adequate exchange between the solvent and non-solvent, followed by a resultant absolute removal of porogen and solvent from the membrane matrices, the prepared membranes were taken out of the water bath and rinsed several instants with water. The entire casting process was carried out in a controlled environmental atmosphere, where temperature and relative humidity were maintained at $25 \pm 1^{\circ} \mathrm{C}$ and $35-40 \%$, respectively. The membranes developed under the invariable casting condition were properly stored in a water-bath. The membranes were categorized on the basis of the difference in the specified compositions, and accordingly designated as: Control-Psf UF; Psf: SWNT-1 UF, Psf:SWNT-2 UF and Psf:C-SWNT-1 
UF, Psf:C-SWNT-2 UF; Psf:DWNT-1 UF, Psf: DWNT-2 UF and Psf:C-DWNT-1 UF, Psf:C-DWNT2 UF; Psf:MWNT-1 UF, Psf:MWNT-2 UF and Psf: C-MWNT-1 UF, Psf:C-MWNT-2 UF.

\subsection{Structural characterization of as-synthesized carboxylated CNTs}

The structural analysis of the synthesized carboxylated CNTs was carried out by using X-ray Photoelectron Spectroscopic technique. A DESA-150 electron analyzer (Staib Instruments, Germany) equipped with $\mathrm{Mg}-\mathrm{K} \alpha \mathrm{X}$-ray source $(1253.6 \mathrm{eV})$ was employed for characterization purpose. The binding energy scale of the spectrometer was calibrated with $\mathrm{Au}-4 \mathrm{f}_{7 / 2}$ photo-peak at a binding energy (B.E.) of $83.95 \mathrm{eV}$. The spectral regions were scanned through several sweeps until a good signal-to-noise ratio was obtained, and then the survey scans were recorded in the range of $0-1000 \mathrm{eV}$. The spectra were recorded as intensity (number of counts per second) versus B.E. The multiplex photo-peaks were subjected to the Gaussian functions to fit the curves, and then peak area as well as the full width at half maximum (FWHM) were determined.

\subsection{Physicochemical characterizations of mixed-matrix UF membranes}

Surface hydrophilic features of the membranes having physicochemical heterogeneities were evaluated through measurements of contact angles by static sessile drop method. A drop shape analyzer (DSA 100 of KRUSS Gmbh, Germany) equipped with DSA 1 v 1.92 software was employed for such evaluation purposes. A $3 \mu \mathrm{L}$ drop of the probe-solvent (water) was deposited using a micro-syringe needle on the membrane surface. The equilibrium contact angle values were measured at the membrane-solvent-air interface with an equal residence time of $60 \mathrm{~s}$. After continuous such measurements being carried out at eight different locations of each membrane surface, the mean contact angle values were determined.

The electrokinetic characteristics of the membranes were evaluated by a ZetaCAD electrokinetic analyzer (CAD Inst., France), which consisted of a quartzcell configuration being capable of holding two flat sheets of each membrane in such a way that the probed membranes remained separated by spacers and their skin layers facing each other create a slit channel for the tangential flow of electrolytic solution across the membranes. The streaming potential, generated by the bidirectional flow of $10^{-3} \mathrm{M} \mathrm{KCl}$ as background electrolyte solution, under applied pressure gradient across the membrane was measured by $\mathrm{Ag} / \mathrm{AgCl}$ electrodes, equipped with the cell. The zeta potential $(\zeta)$ of the membranes was evaluated by the streaming potential values using the Helmholtz-Smoluchowski equation.

For the ease of comparative surface analysis on the reinforcement effect of CNTs of different configurations, mixed-matrix UF membranes derived employing SWNT and MWNT as well as their functionalized counter-parts were selectively exploited. Membranes skin surface topography was characterized by using an atomic force microscope (AFM, Model: SOLVER next, NT-MDT, Russia). The membranes were cut into squared pieces of approximately $1 \mathrm{~cm}^{2}$ and pasted onto a metal substrate. The rectangular cantilever NSG 10 (NT-MDT, Russia) made of $\mathrm{Si}_{3} \mathrm{~N}_{4}$ with a spring constant of $11.8 \mathrm{~N} / \mathrm{m}$, a typical resonance frequency of $240 \mathrm{kHz}$ and a nominal tip apex radius of $10 \mathrm{~nm}$ with a high aspect ratio was employed for scanning purpose. The scanning was done in semicontact mode on a $20 \mu \mathrm{m} \times 20 \mu \mathrm{m}$ area of the membrane in air and at room temperature, with a scanning frequency of $0.1 \mathrm{~Hz}$. The scanned regions were flattened using a second order polynomial to remove the curvatures and slopes from the image and then the resulting best fit was subtracted from it. The surface roughness parameters of the membranes was evaluated by NOVA-P9 software, and calculated from the height profiles of the images in terms of average roughness $\left(R_{\mathrm{a}}\right)$ and root mean square roughness $\left(R_{\mathrm{q}}\right)$. The variations in morphology of the mixed-matrix UF membranes were investigated using a Field Emission Scanning Electron Microscope (FE-SEM, Model: AURIGA, Carl Zeiss, Germany). For the imaging purpose, a piece $\left(0.5 \mathrm{~cm}^{2}\right)$ of the membranes were cut and fractured into smaller sized strips in liquid nitrogen. Then the cross-sectional layers were sputter coated with $\mathrm{Au} / \mathrm{Pd}(60 / 40)$ using a sputter coater (Model No. K550X Emitech), under the optimum conditions (sputtering time: $60 \mathrm{~s}$, sputter current: $30 \mathrm{~mA}$, and tooling factor: 2.3), in order to reduce the effect of electrostatic charging on the electrically non-conductive membrane samples. All the micrographs were recorded at the magnification of $74500 \times$ at an identical acceleration voltage of $5 \mathrm{kV}$, while operating in the secondary electron mode.

The variation in mechanical features was assessed for mixed-matrix UF membranes derived employing 
SWNT and MWNT along with their carboxylated counterparts, in particular. A universal tensile testing machine (Hemetek Techno Instrument, Model: LRX Plus, India) was used by operating at $25^{\circ} \mathrm{C}$ with a relative humidity of $40-50 \%$. For analysis, membrane samples stored in water bath were cut into dumbbell shape with length of $4 \mathrm{~cm}$ and width of $0.6 \mathrm{~cm}$, and these wet membrane specimens were then utilized for the tensile test. A constant deformation rate of $100 \mathrm{~mm} \cdot \mathrm{min}^{-1}$ was maintained during the analysis. The mechanical features like tensile strength (T.S.), and percentage elongation at break (E.B.) were calculated for the membranes in triplicates, using the NEXYGEN plus software, and then averaged out.

\subsection{Evaluation of ultrafiltration performances of mixed-matrix UF membranes}

The efficiency of molecular separation was evaluated by analyzing the rejection behaviours of each UF membrane toward neutral organic solutes, such as $\operatorname{PEG}\left(M_{\mathrm{w}}: 35 \mathrm{kDa}\right)$ and PEO $\left(\mathrm{Mw}_{\mathrm{w}}: 100 \mathrm{kDa}\right)$. The test solutions were prepared by dissolving PEG and PEO in ultra-pure water at a concentration of $200 \mathrm{ppm}$. The membranes with an identical effective area of $14.5 \mathrm{~cm}^{2}$ were employed in a cross-flow filtration unit, being operated under a transmembrane pressure of $1 \mathrm{bar}$ at room temperature. The measurements were repeated with three different coupons of each membrane and the average values were considered. The concentrations of both the feed and product solutions were measured by analyzing the total organic carbon (TOC) content of the samples using a TOC analyzer (ANATOC-II, SGE analytical science, Australia). The percentage rejection of the probe organic solutes $\left(\mathrm{R}_{\mathrm{PEG} / \mathrm{PEO}}\right)$ was determined using Equation (1):

$R_{\text {Solute }}=\frac{C_{\mathrm{F}}-C_{\mathrm{P}}}{C_{\mathrm{F}}} \cdot 100$

where $C_{\mathrm{P}}$ and $C_{\mathrm{F}}$ denote the concentrations of the permeate and feed.

The steady-state solvent flux $\left(J\right.$ in $\mathrm{L} \cdot \mathrm{m}^{-2} \cdot$ day $\left.^{-1}\right)$ was determined by direct as well as replicate measurements of the permeate flow, i.e., the volume of permeate $(V$, in $\mathrm{L}$ ) collected during the time period $(T$, in day), through the membrane area $\left(A\right.$, in $\left.\mathrm{m}^{2}\right)$, and accordingly estimated using Equation (2):

$$
J=\frac{V}{A T}
$$

Prior to all UF test experiments, the membranes were initially subjected to a hydraulic compaction for $1 \mathrm{~h}$ in water at the standard UF test conditions, whilst operated at 1 bar of transmembrane pressure to achieve stabilized performances of the membranes.

\section{Results and discussion \\ 3.1. Analysis of structural characteristics of functionalized CNTs by XPS}

The variations in the extent of functionalization of CNTs have succinctly been investigated through a comparative XPS study, wherein the characteristic peaks belonging to the C-1s spectra of the exploited pristine CNTs, viz. SWNT, DWNT and MWNT are quantitatively contrasted with the C-1s spectra of their functionalized counter-parts, viz. C-SWNT, C-DWNT and C-MWNT. The observed varying functional characteristics, in spite of their exposures under identical oxidative environments, are attributable to the structural heterogeneities of the different CNTs.

The deconvoluted C-1s spectra of SWNT, DWNT and MWNT, exemplified in Figure 1 reveal the presence of intense component peaks at the lowest B.E. of 284.6, 284.7 and $284.6 \mathrm{eV}$ with respective FWHM of 1.6, 1.4 and $1.3 \mathrm{eV}$ (Table 1), which are ascribed to the non-oxygenated $\mathrm{C}$ atoms $(\mathrm{C}-\mathrm{C} / \mathrm{C}=\mathrm{C} / \mathrm{C}-\mathrm{H})$ of the graphitic structures [24]. The broader peaks appearing at higher B.E. of $286.0,286.1$ and $286.6 \mathrm{eV}$ with FWHM of 2.4, 2.1 and $2.2 \mathrm{eV}$, respectively could be attributed to the $\mathrm{C}$ atoms of the residual $-\mathrm{C}-\mathrm{OH}$ functionalities, resulting out of the acid treatment to remove the transition metal catalyst particles during post-synthesis purification of as-received CNTs [24]. In the pristine MWNT, the peak appearing at B.E. of $285.4 \mathrm{eV}$ (FWHM: $1.6 \mathrm{eV}$ ) corresponds to the defects on the nanotubes' structure [25]. The surfacing of such characteristic peak implies the enhanced probability of defects in the nanotubes' structures with the increase in number of walls, and thus the resultant distortion in $\mathrm{C}-\mathrm{C}$ chains is noticed to be significant in MWNT, unlike DWNT and SWNT. The least intense peaks located at 288.4, 288.1 and $288.9 \mathrm{eV}$ with respective FWHM of 4.5, 2.4 and $6.2 \mathrm{eV}$ are assigned to the residual $\mathrm{O}-\mathrm{C}=\mathrm{O}$ segments of the pristine SWNT, DWNT and MWNT, respectively [24].

The XPS spectra represented in Figure 2 enlighten the variations observed in the functionalized nanostructures of exploited CNTs, wherein the controlled functionalization seems to introduce substantial 

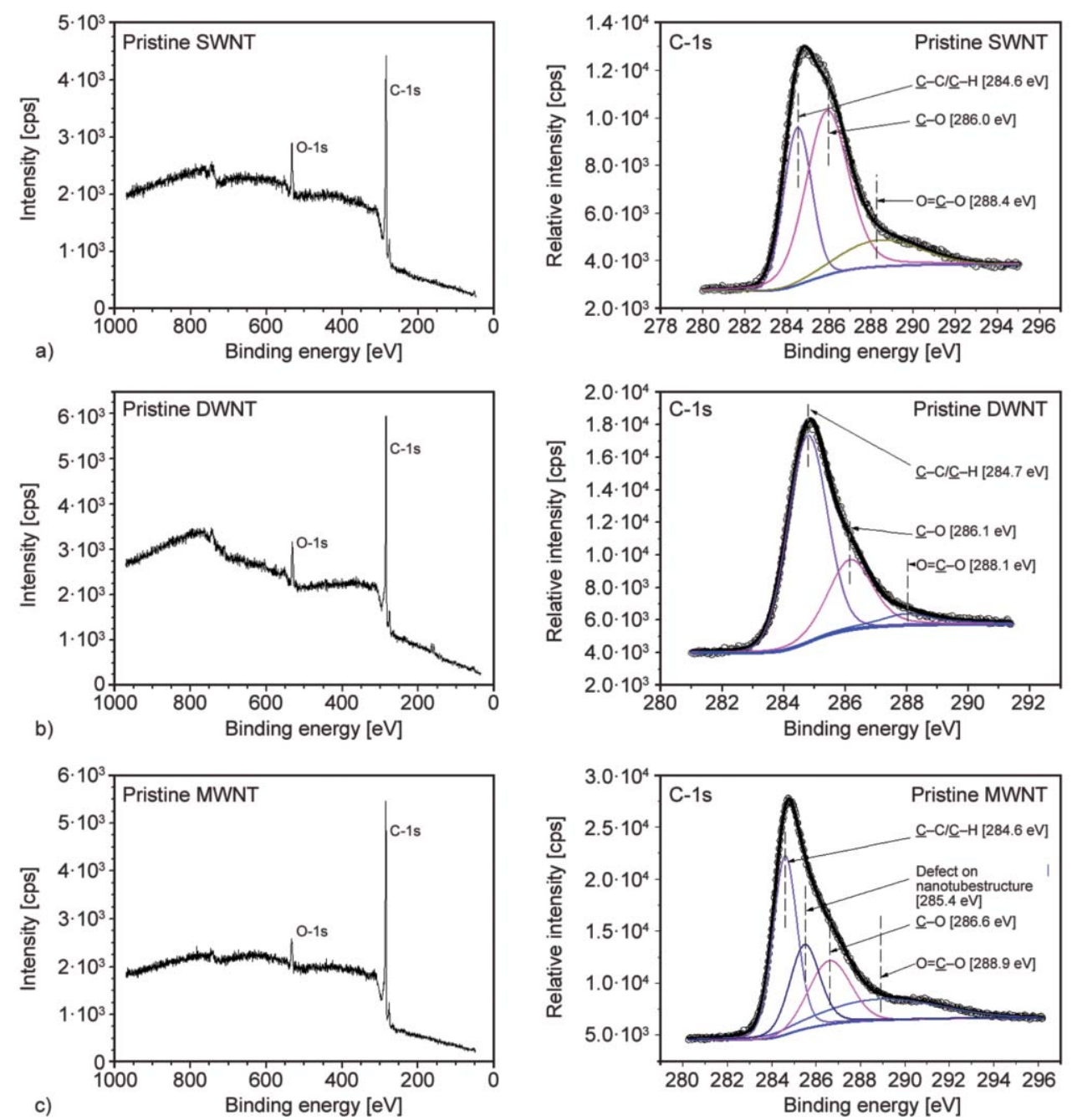

Figure 1. Survey scan spectra and high resolution C-1s photoelectron spectra of pristine CNTs: a) SWNT, b) DWNT, and c) MWNT, (line with bullets: experimental data; solid line: curve fit of the experimental data)

oxygen rich functionalities at different proportions. The deconvoluted C-1s spectra of C-SWNT, CDWNT and C-MWNT (Figure 2) reveal the emergence of distinctive peaks, amongst which the peaks ascribed to the non-oxygenated $\mathrm{C}$ atoms of graphitic structures appear at 284.4, 284.8 and $284.6 \mathrm{eV}$ with

Table 1. The summary of curve fitting of C-1s XPS spectra of pristine SWNT, DWNT and MWNT

\begin{tabular}{|l|c|c|c|}
\hline \multirow{2}{*}{$\begin{array}{c}\text { Codes of } \\
\text { pristine CNTs }\end{array}$} & \multicolumn{3}{|c|}{ C-1s } \\
\cline { 2 - 4 } & $\begin{array}{c}\text { BE } \\
{[\mathbf{e V}]}\end{array}$ & $\begin{array}{c}\text { FWHM } \\
{[\mathbf{e V}]}\end{array}$ & $\begin{array}{c}\text { Peak area } \\
{[\%]}\end{array}$ \\
\hline \multirow{3}{*}{ SWNT } & 284.6 & 1.6 & 30.8 \\
& 286.0 & 2.4 & 54.2 \\
& 288.4 & 4.5 & 14.9 \\
\hline \multirow{3}{*}{ DWNT } & 284.7 & 1.4 & 51.3 \\
& 286.1 & 2.1 & 42.4 \\
& 288.1 & 2.4 & 6.3 \\
\hline \multirow{3}{*}{ MWNT } & 284.6 & 1.3 & 36.4 \\
& 285.4 & 1.6 & 22.3 \\
& 286.6 & 2.2 & 21.6 \\
& 288.9 & 6.2 & 19.7 \\
\hline
\end{tabular}

FWHM of 2.1, 1.5 and $1.4 \mathrm{eV}$, respectively (Table 2). The extent of graphitic carbons in the functionalized CNTs is observed to vary on carboxylation. The rise in concerned peak area and FWHM for C-SWNT implies that, shear stresses induced by ultrasonication prior to the functionalization followed by stirring during the course of functionalization might have debundled few nanotubes, and thereby enhanced the configurational entropy of the reactive system [26]. However, despite of the similar effect persuaded by shear stresses in the reactive systems of C-DWNT and C-MWNT, the observed variations in FWHM and peak area for their concerned peaks may sensibly be considered as synchronized compensations by the varying contributions of $\mathrm{C}$ atoms belonging to the tethered oxygenated functionalities, which seem to be visually contrasting with CSWNT. Such behaviours may also get influenced by the differential reactivity of the CNTs under oxidative environment. 

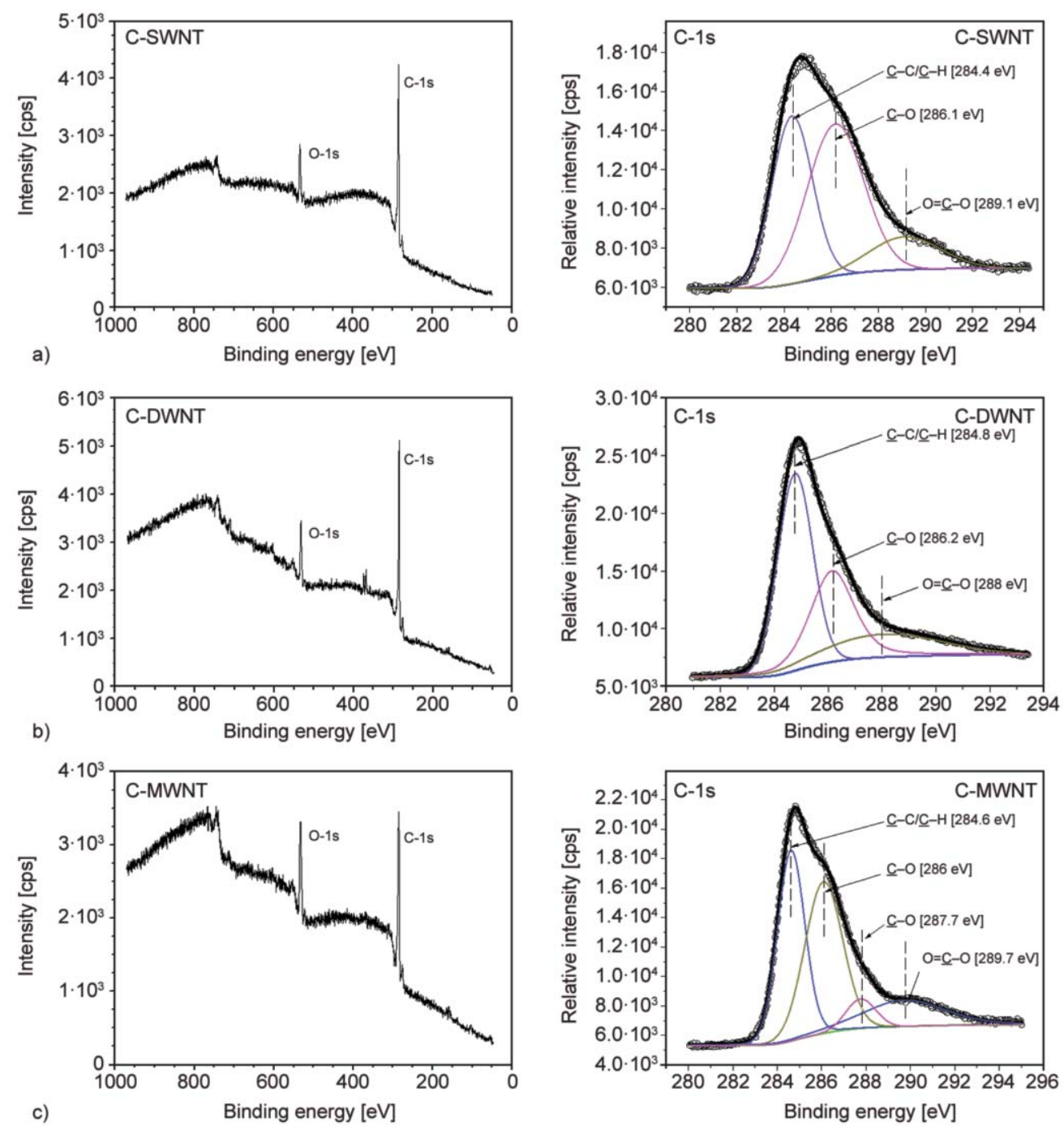

Figure 2. Survey scan spectra and high resolution C-1s photoelectron spectra of functionalized CNTs: a) C-SWNT, b) C-DWNT, and c) C-MWNT, (line with bullets: experimental data; solid line: curve fit of the experimental data)

The characteristic peaks appearing at higher B.E. of 286.1, 286.2 and $286.0 \mathrm{eV}$ (Figure 2) are referred to the $\mathrm{C}$ atoms of $-\mathrm{C}-\mathrm{OH}$ functionalities belonging to C-SWNT, C-DWNT and C-MWNT, respectively. A comparison of the respective peak areas (Table 2)

Table 2. The summary of curve fitting of C-1s XPS spectra of functionalized CNTs: C-SWNT, C-DWNT and C-MWNT

\begin{tabular}{|l|c|c|c|}
\hline $\begin{array}{c}\text { Codes of } \\
\text { functionalized } \\
\text { CNTs }\end{array}$ & \multicolumn{3}{|c|}{ C-1s } \\
\cline { 2 - 4 } & $\mathbf{B E}$ & $\begin{array}{c}\text { FWHM } \\
{[\mathbf{e V}]}\end{array}$ & $\begin{array}{c}\text { Peak area } \\
{[\mathbf{e V}]}\end{array}$ \\
\hline \multirow{3}{*}{ C-SWNT } & 284.4 & 2.1 & 39.1 \\
& 286.1 & 2.8 & 47.8 \\
& 289.1 & 3.6 & 13.1 \\
\hline \multirow{3}{*}{ C-DWNT } & 284.8 & 1.5 & 48.1 \\
& 286.2 & 1.9 & 33.1 \\
& 288.0 & 5.1 & 18.8 \\
\hline & 284.6 & 1.4 & 36.9 \\
C-MWNT & 286.0 & 2.0 & 39.9 \\
& 287.7 & 1.6 & 5.1 \\
& 289.7 & 4.3 & 17.1 \\
\hline
\end{tabular}

signifies that the extent of such weakly oxidized functionality gets reduced by 6.4 and $9.3 \%$ on carboxylation of SWNT and DWNT, respectively. This can be ascribed to further emergences of oxidized functionalities in the structures of C-SWNT and C-DWNT. On functionalization of SWNT, the peak corresponded to the strongly oxidized carboxylic $(-\mathrm{COOH})$ functionality appears at higher B.E. of $289.1 \mathrm{eV}$ in C-SWNT. The emergence of a third constituent peak at B.E. of $288.0 \mathrm{eV}$ in C-DWNT, originating from the functionalization of DWNT is assigned to the - $\mathrm{COOH}$ functionality, which compensates the decline in density of - $\mathrm{C}-\mathrm{OH}$ functionality. However, the carboxylation on MWNT seems to be controlled in a different manner as the extent of presence of $-\mathrm{C}-\mathrm{OH}$ functionality is observed to get increased in C-MWNT by $18.3 \%$ as compared to MWNT. It is projected by earlier research studies that, the SWNTs existing in bundling morphology experience strong attractive 
van der Waals forces and $\pi$ - $\pi$ interactions between the hydrophobic graphitic walls [26, 27], and thus opt to get oxidized at the nanotube end-tips as well as the outer layers of the bundles. However, the MWNTs existing in clustering morphology prefer their nanotube end-tips and the larger number of spatially available defect sites to get oxidized at the onset, and then allow the oxidation to progress from the outermost graphite layer to the nanotube core [26]. The deconvoluted C-1s spectra of C-MWNT reveal the presence of two more constitutional peaks that appear at 287.7 and $289.7 \mathrm{eV}$, attributable to the $\mathrm{C}$ of carbonyl $(>\mathrm{C}=\mathrm{O})$ and $-\mathrm{COOH}$ functionalities, respectively. This subsequently corroborates the fact of preferential enrichment of MWNTs with oxygen rich functionalities. Our study indicates that the trend of favoured oxidation is MWNT $>$ DWNT $>$ SWNT, which signifies that the extent of carboxylation depends on configurations of CNTs and so the available surface area plays a decisive role in providing effective interaction sites ensuring better reactivity of the CNTs.

\subsection{Study of hydrophilic features of mixed-matrix UF membranes}

Analytic assessment of hydrophilicity has been made with respect to the probe solvent - water to evaluate the function of pristine CNTs (SWNT, DWNT and MWNT) and carboxylated CNTs (C-SWNT, CDWNT and C-MWNT) as nanoadditive in modifying the surface chemistry of mixed-matrix UF membranes. Considering the Control-Psf UF with contact angle of $70.7 \pm 0.6^{\circ}$ (Figure 3a), the same factor is observed to decrease moderately on reinforcement of pristine CNTs at lesser concentration, such as Psf: SWNT-1 UF: $63.4 \pm 0.5^{\circ}$, Psf:DWNT-1 UF: $61.5 \pm 0.6^{\circ}$, and Psf:MWNT-1 UF: $63.7 \pm 0.5^{\circ}$. The decline in the contact angles could be owing to the mere presence

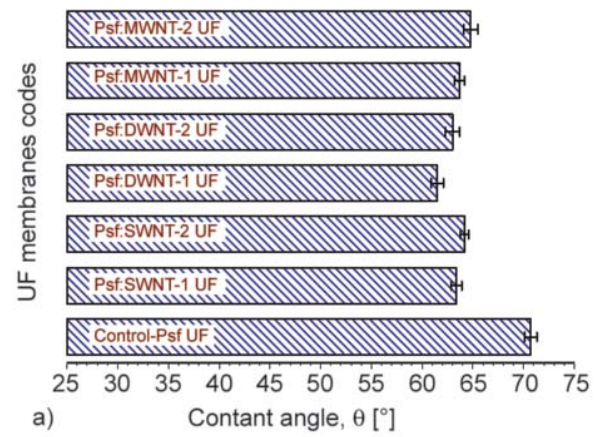

of oxygen containing impurities residing over the CNT surfaces. The organic dispersion medium, i.e., amide solvent - NMP consisting of an alkyl substituent on $\mathrm{N}$ atom gets resonance stabilized, and so acquires a high electronic dipole-moment $(\mu=4.1 \mathrm{D})$ [28]. The high polarity as well as optimal geometries (appropriate bond lengths and bond angles) thus enables the aprotic solvent molecules of NMP to undergo favourable dipolar interactions with the CNTs, during dispersion of the latter in the former [29]. In such condition, the nanotube-solvent (CNT-NMP) interaction is supposed to prevail over the equilibrated interaction of solvent-solvent (NMP-NMP) and nanotube-nanotube (CNT-CNT), ensuring enhancement in repulsive van der Waals forces between the CNTs. This resultantly leads to thermodynamically stable dispersions of the exploited pristine CNTs in NMP at lower concentration, by minimizing the enthalpy of mixing $\left(\Delta H_{\text {mix }}\right)$ and enhancing the configurational entropy $[21,30]$. However, it is observed with enhanced reinforcement of CNTs, resulting increase in the solution viscosity that the contact angle values increase slightly, i.e., Psf:SWNT-2 UF: $64.2 \pm 0.4^{\circ}$, Psf:DWNT-2 UF: $63.0 \pm 0.7^{\circ}$ and Psf:MWNT-2 UF: $64.8 \pm 0.7^{\circ}$. This reflects an escalation of adverse hydrophobicity, which could be because of a cohesive contribution of enhanced attractive van der Waals forces between the hydrophobic walls of CNTs at higher concentration and inherent hydrophobic polymeric segments of Psf.

The variations in hydrophilic characters (Figure 3b), brought by the carboxylated CNTs is more pronounced as compared to the case of pristine CNTs in altering the membranes' surface chemistry. The mixed-matrix UF membranes derived utilizing CSWNT, C-DWNT and C-MWNT exert distinctive decline in contact angles, i.e., $60.9 \pm 0.9$ to $54.2 \pm 1.1^{\circ}$, $59.1 \pm 1.2$ to $55.3 \pm 0.9^{\circ}$ and $58.1 \pm 1.2$ to $50.9 \pm 1.4^{\circ}$,

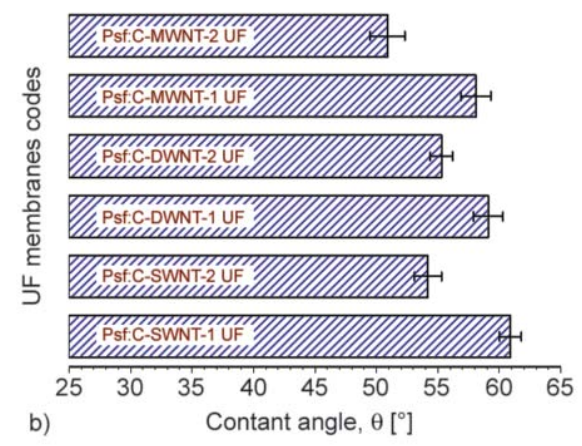

Figure 3. Variation in hydrophilic characteristics, a) Control-Psf UF and pristine CNTs reinforced mixed-matrix UF membranes, and b) carboxylated CNTs reinforced mixed-matrix UF membranes 
respectively, on enhanced reinforcement of the carboxylated CNTs. The presence of electron-withdrawing $-\mathrm{COOH}$ groups on the functionalized CNTs is supposed to reduce the charge density of the nanotubes through electron transfer from the valence bands of nanotubes to the tethered functionalities [31], which influences the dipolar interaction between the carboxylated CNTs and NMP. Other secondary interaction like intermolecular $\mathrm{H}$-bonding between the carboxylated CNTs and NMP also gets facilitated under the modulated chemical environment. Such favourable CNT-NMP enthalpic interaction is presumed to reduce the enthalpic interaction of CNTCNT, resulting minimization of $\Delta H_{\text {mix }}$, and subsequent enhancement of dispersion stability. Contrary to the pristine CNTs, the dispersion stability of carboxylated CNTs gets facilitated through the formation of finer chemical sheath by the plenty of additive (PVP) molecules in presence of the base polymer, Psf [32]. Thus, the carboxylation stimulates the CNTs to achieve higher configurational entropy, and intensely affects the solution chemistry to form a better stable dispersion as compared to the pristine ones.

The Hansen solubility parameter (HSP), being important material property to illustrate the physicochemical compatibility of CNTs gets defined through the addition of three different contributing factors, $v i z$. dispersive interactions $\left(\delta_{\mathrm{D}}\right)$, polar interactions $\left(\delta_{\mathrm{P}}\right)$ and hydrogen bonding interactions $\left(\delta_{\mathrm{H}}\right)$ [33]. The dispersive Hansen parameter, $\delta_{\mathrm{D}}$ has an inverse relationship with the diameter of CNTs, since the former gets decided by the extent of van der Waals forces [33]. Therefore, it seems rational to mention that $\delta_{\mathrm{D}}$ becomes increasingly higher from pristine MWNTs to DWNTs, and further to SWNTs. However, it is expected that the said factor gets minimized in carboxylated CNTs. In contrast to the variation in $\delta_{\mathrm{D}}$, the other Hansen parameters, $\delta_{\mathrm{P}}$ and $\delta_{\mathrm{H}}$ tend to increase on carboxylation, following the extent of enrichment of the CNTs with oxygen rich functionalities, as elucidated through XPS analysis. Since, the dispersion stability is proportionally related to the closeness of HSP of the nanotube to that of solvent, thus, individual proximity of the factors $\delta_{\mathrm{D}}, \delta_{\mathrm{P}}$ and $\delta_{\mathrm{H}}$ of carboxylated CNTs to the factors of solvent $-\mathrm{NMP}\left(\delta_{\mathrm{D}}=18.0, \delta_{\mathrm{P}}=12.3\right.$, and $\delta_{\mathrm{H}}=$ $7.2 \mathrm{MPa}^{1 / 2}$ ) tune the nanotubes' physicochemical compatibility [28]. The aforementioned variations in hydrophilic features of the mixed-matrix UF membranes substantially signify the effectiveness of facilitated dispersion that gets manifested more in CMWNT as compared to the C-SWNT.

It seems imperative to mention that during non-solvent induced phase inversion process, unlike the case of pristine CNTs, the spontaneous migrations of the stimulated nanoadditives, i.e., carboxylated CNTs toward the skin regions of the asymmetric membranous structures get favoured owing to the more affinity of them toward the polar-protic nonsolvent, water over the polar-aprotic solvent, NMP, resulting a facilitated distribution by reducing the interfacial energy [34]. These mixed-matrix UF membranes further experience improved interfacial compatibility between the nanoadditives and the host polymer matrix through favoured intermolecular $\mathrm{H}$ bonding interactions involving the $-\mathrm{COOH}$ groups of carboxylated CNTs and sulfone $(\mathrm{O}=\mathrm{S}=\mathrm{O})$ groups of Psf [34]. Amongst the three different kinds of functionalized CNTs, the C-MWNT thus relevantly becomes more persuasive in comparison of the $\mathrm{C}$ DWNT and C-SWNT to tune the hydrophilicity of the membranes; the fact which also gets substantiated by the enlightened variations in enrichment of oxygen rich functionalities of carboxylated CNTs, as discussed during XPS study.

\subsection{Study of electrokinetic characteristics of mixed-matrix UF membranes}

Electrokinetic features of the probed UF membranes, determined by tangential streaming potential measurements with respect to $10^{-3} \mathrm{M} \mathrm{KCl}$ solution are represented in Figure 4. The Control-Psf UF with a matrix devoid of any dissociable functionality being capable of creating surface charges showed a $\zeta$ of $-20.4 \pm 0.4 \mathrm{mV}$, which is attributed to the effect of specific adsorptions of $\mathrm{Cl}^{-}$ions from the electrolyte solution on the hydrophobic membrane surface [35]. However, the investigated mixed-matrix UF membranes consist of different weak or strong sources of charges, which arise due to the residual weaker acidic groups $(-\mathrm{OH})$ on the surface of CNTs or the covalently attached stronger acidic groups $(-\mathrm{COOH})$ of the functionalized CNTs. Therefore, the preference of a mixed-matrix enriched with dissociable functionalities seems to be capable of surpassing the tentative ionic adsorptions by the intrinsically hydrophobic core matrix since the charge carrying sites induce surface conductivity in the membranes [36], and through their variant distributions alter the electrokinetic features as well. 


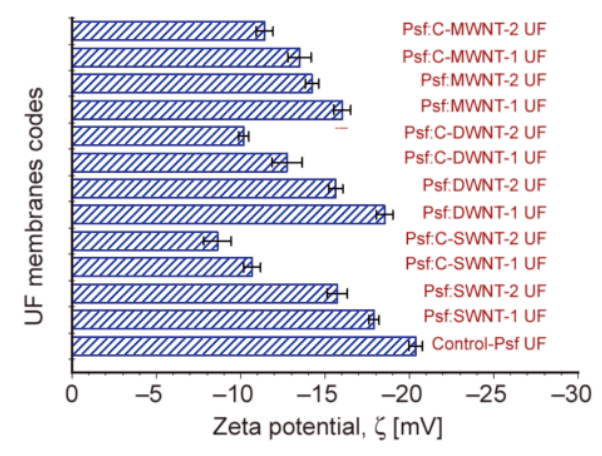

Figure 4. Variation in electrokinetic features of Control-Psf and mixed-matrix UF membranes

The occurrence of ionic adsorption gets substantially reflected through the variations in $\zeta$ of the mixedmatrix UF membranes, such as $-17.9 \pm 0.3 \mathrm{mV}$ for Psf:SWNT-1 UF to $-15.7 \pm 0.6 \mathrm{mV}$ for Psf:SWNT-2 UF. Similar descending trend is also observed in the case of DWNT and MWNT reinforced mixed-matrix UF membranes, as there $\zeta$ changes from $-18.5 \pm 0.5 \mathrm{mV}$ for Psf:DWNT-1 UF to $-15.6 \pm 0.4 \mathrm{mV}$ for Psf:DWNT$2 \mathrm{UF}$, and $-16.1 \pm 0.5 \mathrm{mV}$ for Psf:MWNT-1 UF to $-14.2 \pm 0.4 \mathrm{mV}$ for Psf:MWNT-2 UF. The decline in the negative $\zeta$ values of the mixed-matrix UF membranes as compared to the Control-Psf UF substantiates that, the inclination toward severe ionic adsorption gets comparatively overcompensated by the occurred electrokinetic events influenced through the modified compositions of electrochemical double layers in the formers.

The influence of electrochemical double layers' composition is observed to become more pronounced on reinforcement of carboxylated CNTs within the core Psf matrix. The enhanced reinforcements of carboxylated CNTs markedly elevate the skin regions' conductivities of the respective membranes. The descending trend of negative $\zeta$ values for the C-SWNT, C-DWNT and C-MWNT reinforced mixed-matrix UF membranes is found to be $-10.7 \pm 0.5$ to $-8.6 \pm 0.8 \mathrm{mV}$ for Psf:C-SWNT-1 UF and Psf:C-SWNT-2 UF, $-12.7 \pm 0.9$ to $-10.2 \pm 0.3 \mathrm{mV}$ for Psf:C-DWNT-1 UF and Psf:C-DWNT-2 UF, and then $-13.5 \pm 0.7$ to $-11.4 \pm 0.5 \mathrm{mV}$ for Psf:C-MWNT-1 UF and Psf:CMWNT-2 UF. This variance is owing to the dragging of the tangentially driven counter-ions from the hydrodynamic plane of shear to the bulk of the membranes' skin region enriched with dissociable functionalities. The effective streaming currents of the membranes are thus supposed to get reduced to some extent by such diffusive back flow of the streaming current [37]. This tends to be get favoured by both distribution of carboxylated CNTs at the skin regions, rather than the bulk regions of the membranes and spatial orientation of $-\mathrm{COOH}$ sites in the membrane-air or membrane-solvent interface.

\subsection{Analysis of surface topography and morphology of mixed-matrix UF membranes}

The topographical features of the skin surfaces of selective mixed-matrix UF membranes, probed through AFM delineate the role of functionalization in amending the superior distribution of the carboxylated CNTs as compared to the respective pristine counter-parts within polymeric host matrix. The roughness parameters, $R_{\mathrm{a}}$ and $R_{\mathrm{q}}$ (Table 3 ) enhance significantly from Control-Psf UF $(8.9 \pm 0.4$ and $11.6 \pm 0.5 \mathrm{~nm})$ to Psf:SWNT-1 UF $(14.6 \pm 0.5$ and $18.9 \pm 0.3 \mathrm{~nm})$, Psf:C-SWNT-1 UF $(12.5 \pm 0.2$ and $16.1 \pm 0.4 \mathrm{~nm})$ as well as Psf:MWNT-1 UF (14.3 \pm 0.6 and $19.9 \pm 0.3 \mathrm{~nm})$, Psf:C-MWNT-1 UF $(10.4 \pm 0.5$ and $15.4 \pm 0.7 \mathrm{~nm})$. The 3D AFM images of skin surfaces of the Psf:SWNT-1 UF (Figure 5a) and Psf:C-SWNT-1 UF (Figure 5b) distinctively signify the emergence as well as extent of aggregated features in the former category, and minimization of such in the latter class of membrane. A similar change in skin surface topography is also noticed in the 3D AFM images of the Psf:MWNT-1 UF (Figure 5c) and Psf:C-MWNT-1 UF (Figure 5d). The observed variations could be attributed to the enhanced effective surface area for the mixed-matrix UF membranes on impregnation of both pristine as well as carboxylated CNTs, irrespective of configurational variations. However, the enhancement in surface area is more pronounced for the former nanoadditives in contrary to the latter, i.e., the carboxylated SWNTs and MWNTs, since both

Table 3. Surface roughness parameters of UF membranes: Control-Psf UF, Psf:SWNT-1 UF, Psf:C-SWNT-1 UF, Psf:MWNT-1 UF and Psf:C-MWNT-1 UF

\begin{tabular}{|c|c|c|c|c|c|}
\hline \multirow{2}{*}{$\begin{array}{c}\text { Roughness } \\
\text { parameters }\end{array}$} & Membranes codes \\
\cline { 2 - 6 } & Control-Psf UF & Psf:SWNT-1 UF & Psf:C-SWNT-1 UF & Psf:MWNT-1 UF & Psf:C-MWNT-1 UF \\
\hline$R_{\mathrm{a}}[\mathrm{nm}]$ & $8.9 \pm 0.4$ & $14.6 \pm 0.5$ & $12.5 \pm 0.2$ & $14.3 \pm 0.6$ & $10.4 \pm 0.5$ \\
\hline$R_{\mathrm{q}}[\mathrm{nm}]$ & $11.6 \pm 0.5$ & $18.9 \pm 0.3$ & $16.1 \pm 0.4$ & $19.9 \pm 0.3$ & $15.4 \pm 0.7$ \\
\hline
\end{tabular}



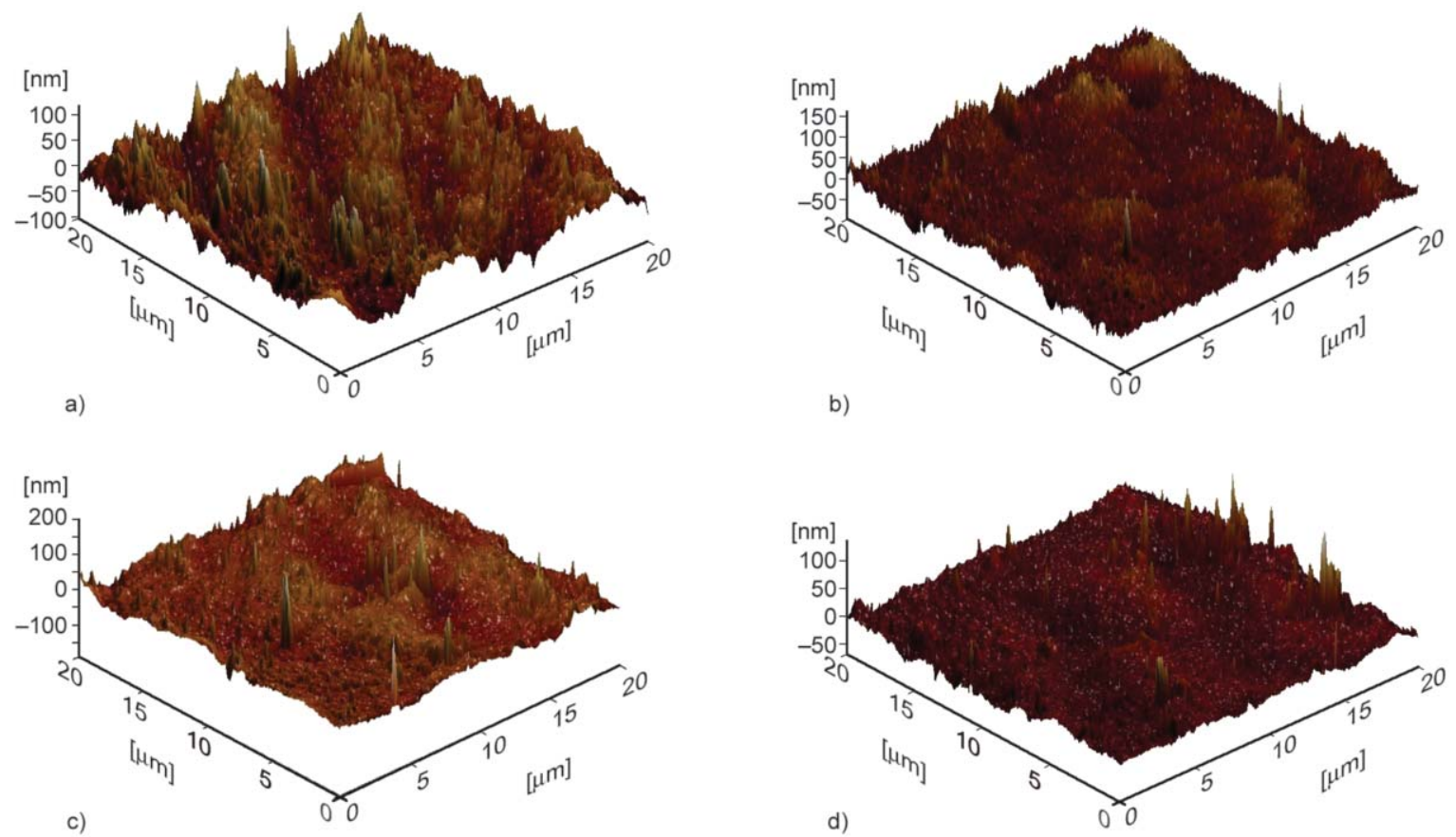

Figure 5. 3D AFM images showing the impressions of aggregation in mixed-matrix UF membranes skin surfaces, a) Psf:SWNT-1 UF, b) Psf:C-SWNT-1 UF, c) Psf:MWNT-1 UF and d) Psf:C-MWNT-1 UF

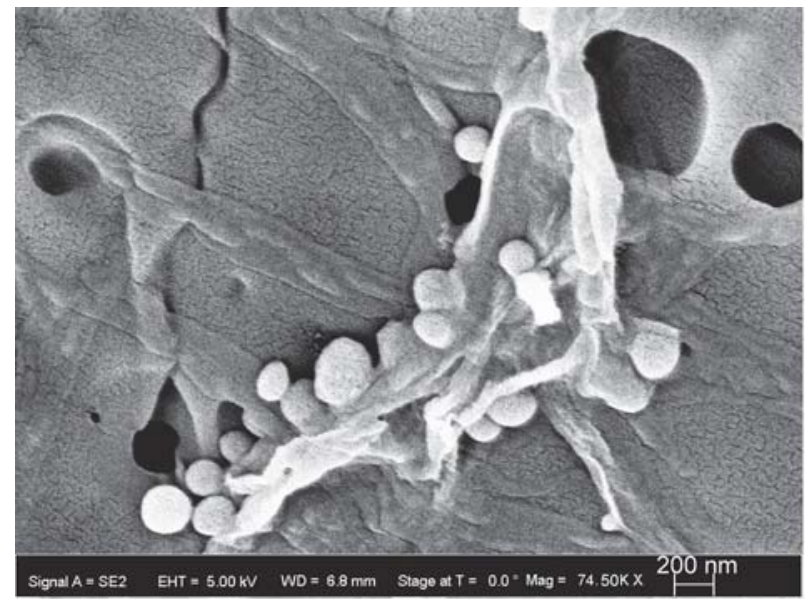

a)

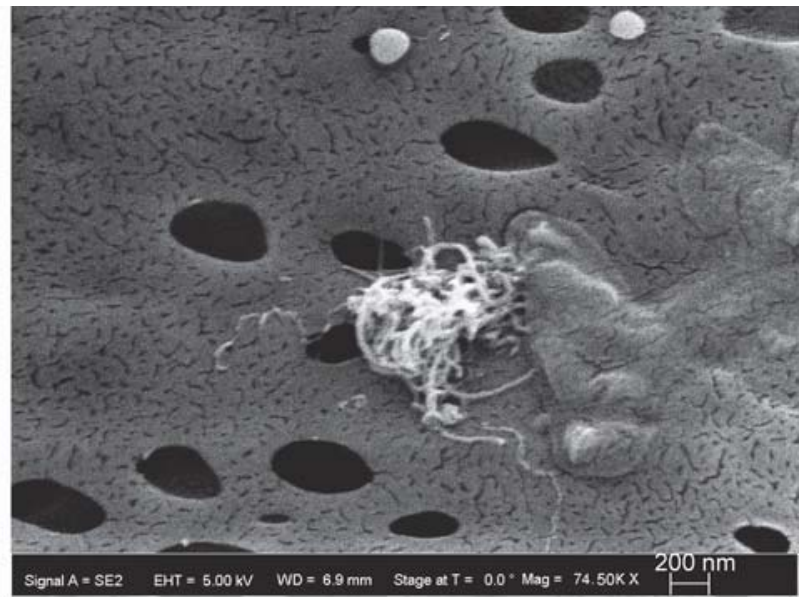

b)

Figure 6. Representing the aggregated features of a) pristine SWNTs and b) pristine MWNTs in the substructural porous regions of respective mixed-matrix UF membranes, Psf:SWNT-1 UF and Psf:MWNT-1 UF, respectively, acquired by FE-SEM at the magnification of $74500 \times$

$R_{\mathrm{a}}$ and $R_{\mathrm{q}}$ values decline on impregnation of the carboxylated CNTs at similar concentration. This signifies the enhanced dispersion induced facilitated distribution of the functionalized CNTs, as discussed in earlier sections. The extent of decline in surface roughness values for both classes of the mixed-matrix UF membranes further implies that C-MWNT plays a superior role over the C-SWNT.

The FE-SEM images of the mixed-matrix UF membranes derived using the pristine SWNTs and MWNTs (Figure 6) reveal that both interior sub-structural regions of the asymmetric porous structures consist of aggregated features, i.e., the presence of clusters of
CNTs. The higher inter-particle attractive force induces flocculation of the pristine CNTs, which further enhances with progressive concentration. This signifies the concerned consequences of noxious dispersion of the non-functionalized CNTs in the present dispersion medium.

\subsection{Study of mechanical features of mixed-matrix UF membranes}

The reinforcement effects of the pristine SWNTs and MWNTs in contrast to their carboxylated counterparts, as compared by analyzing the mechanical features of resultant mixed-matrix UF membranes in 
Table 4. Mechanical properties of UF membranes: Control-Psf UF, Psf:SWNT-1 UF, Psf:SWNT-2 UF, Psf:MWNT-1 UF and Psf:MWNT-2 UF

\begin{tabular}{|l|c|c|c|c|c|}
\hline \multirow{2}{*}{$\begin{array}{c}\text { Mechanical } \\
\text { properties }\end{array}$} & \multicolumn{5}{|c|}{ Membranes codes } \\
\cline { 2 - 6 } & Control-Psf UF & Psf:SWNT-1 UF & Psf:SWNT-2 UF & Psf:MWNT-1 UF & Psf:MWNT-2 UF \\
\hline T.S. $[\mathrm{MPa}]$ & $2.94 \pm 0.15$ & $3.52 \pm 0.21$ & $3.29 \pm 0.16$ & $3.52 \pm 0.18$ & $3.34 \pm 0.23$ \\
\hline Total elongation [\%] & $24.83 \pm 1.62$ & $34.27 \pm 1.51$ & $21.08 \pm 1.72$ & $37.67 \pm 1.56$ & $34.12 \pm 1.12$ \\
\hline
\end{tabular}

Table 5. Mechanical properties of UF membranes: Psf:C-SWNT-1 UF, Psf:C-SWNT-2 UF, Psf:C-MWNT-1 UF and Psf:C-MWNT-2 UF

\begin{tabular}{|l|c|c|c|c|}
\hline \multirow{2}{*}{ Mechanical properties } & \multicolumn{4}{|c|}{ Membranes codes } \\
\cline { 2 - 5 } & Psf:C-SWNT-1 UF & Psf:C-SWNT-2 UF & Psf:C-MWNT-1 UF & Psf:C-MWNT-2 UF \\
\hline T.S. $[\mathrm{MPa}]$ & $3.43 \pm 0.18$ & $3.45 \pm 0.12$ & $3.46 \pm 0.24$ & $3.41 \pm 0.21$ \\
\hline Total elongation [\%] & $36.60 \pm 1.12$ & $35.94 \pm 1.08$ & $39.98 \pm 1.34$ & $34.07 \pm 1.46$ \\
\hline
\end{tabular}

wet condition are represented in Table 4 and 5. This particularly illustrates the predominant role of dispersion stability of the nanoadditives as well as the extent of interfacial compatibility between nanoadditives, i.e., CNTs or carboxylated CNTs and Psf in varying the interfacial stress transfer within membranous matrices.

It is observed that, the CNT as well as carboxylated CNT reinforced UF membranes generally exhibit superior T.S. (tensile strength) as compared to the Control-Psf UF membrane [13]. On enhanced reinforcement of pristine CNTs, the T.S. of the corresponding mixed-matrix UF membranes decreases by $6.5 \%$ for SWNT and $5.1 \%$ for MWNT (Table 4), however, the same factor remains more or less unaffected for the C-SWNT and C-MWNT (Table 5). This is attributable to the effect of facilitated dispersion of the carboxylated CNTs over the pristine ones in the dope solutions. It is assumed that, the pristine CNTs experiencing strong van der Waals forces may transform from a microscopic dispersion to a macroscopic dispersion at their higher concentration in the organic medium, whereas the carboxylation enforces the CNTs to attain nanoscopic dispersion [38]. Therefore,

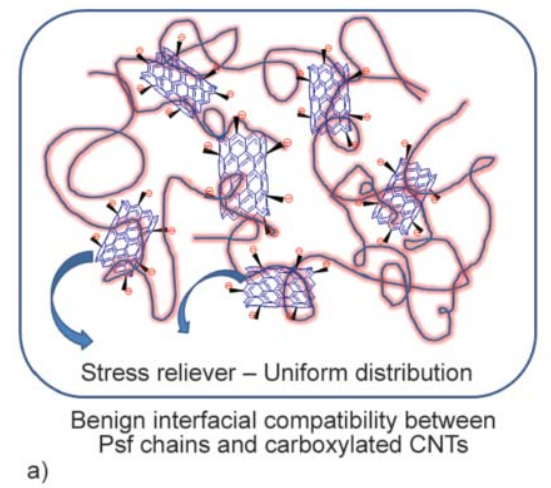

the well dispersed carboxylated CNTs that play the role of stress reliever (Figure 7a) during their uniform distribution by means of superior and stronger interfacial chemical compatibility with the Psf, effectively transfer the mechanical stress through the interface of stretched chains of the Psf and the residing functional groups of the CNTs, even at higher concentration [23]. In contrary, the pristine CNTs exerting poor interfacial chemical compatibility with the polymeric host matrix and further owing to the aggregated structures at higher concentration behave like stress concentrator (Figure 7b), and in consequence become unable to sufficiently relieve the stress of polymeric chains through the interface [39].

It is noticed that the E.B. of the mixed-matrix UF membranes decreases on enhanced reinforcement of the nanoadditives, which seems to be an outcome of the membranes reduced elasticity with progressive reinforcement of the pristine CNTs or carboxylated CNTs into the Psf matrices. A comparison of the variation in E.B. (Table 4) for the mixed-matrix UF membranes of former category reflects that, the detrimental loss of elasticity on enhanced reinforcement of the nanoadditive is significantly pronounced for the

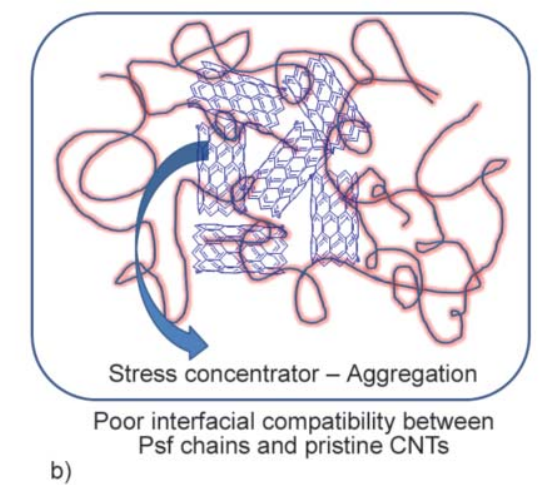

b)

Figure 7. Schematics of variation in the interfacial compatibility of a) carboxylated CNTs as stress reliever, and b) pristine CNTs as stress concentrator with host Psf matrices 
SWNTs, and the E.B. even falls below those of the Control-Psf UF at higher concentration; however the elasticity gets remarkably restored while carboxylated SWNTs are reinforced, even at higher concentration (Table 5). In the case of mixed-matrix UF membranes derived by reinforcing MWNTs or C-MWNTs, the similar behaviour of decline in elasticity gets observed on enhanced reinforcement of the nanoadditives. Indeed, the mixed-matrix UF membrane reinforced with C-MWNTs at lower concentration exhibits the highest elastic response, which further corroborates the superiority of the nanoadditive with higher available surface area and configurational entropy to surpass the tentative aggregation effects. This vividly enlightens the efficacy of functionalization of CNTs in fine-tuning the interfacial chemical environments existing between the polymeric host matrix and the nanoadditives, and thereby the membranes' mechanical features. Although, the extent of variations in the present cases signifies that the approach of carboxylation to enhance the interfacial compatibility between the reinforced CNTs and polymeric host matrix might have intrinsically created defects on the nanotubes' structure further to generate active functional groups, which adequately reduces the tensile strength of the nanotubes leading to lessening of the overall effectiveness in the mixedmatrix UF membranes.

\subsection{Investigation of mixed-matrix UF membranes solute rejection and solvent flux behaviours}

The macroscopical features of the mixed-matrix UF membranes, presented in terms of \%SR and PWP, measured under a steady operating condition are depicted in Figure 8 and 9, respectively, which substantiate the anticipated superior potency of functionalized CNTs as nanoadditives in stimulating the mem-

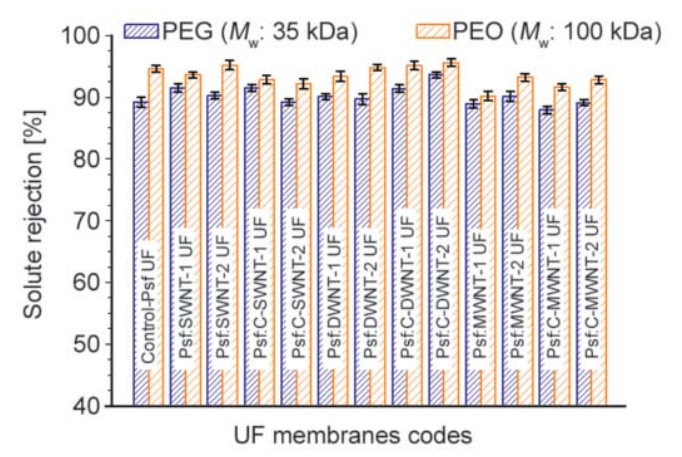

Figure 8.Variation in solute rejection behaviours of UF membranes toward the organic solutes, PEG and PEO

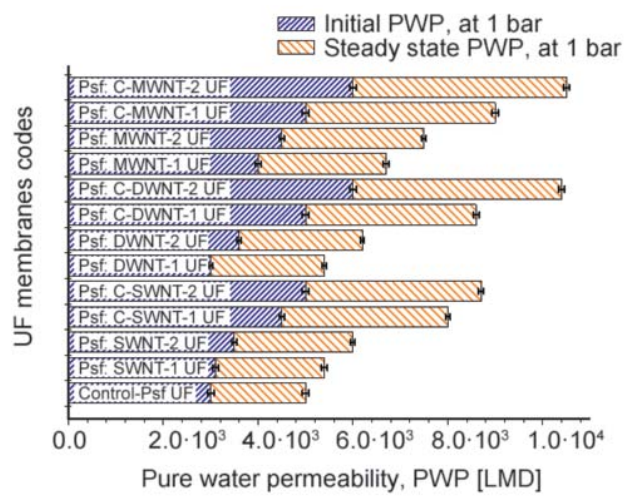

Figure 9. Variation in pure water permeability of UF membranes under steady operating conditions

branes' physicochemical characteristics. The \%SR values for the PEG and PEO are found to be $89.2 \pm 0.8$ and $94.6 \pm 0.6 \%$, respectively for the Control-Psf UF. The depiction in Figure 8 shows that the mixed-matrix UF membranes derived by reinforcing the pristine CNTs as nanoadditive maintain a similar rejection trend as like the Control-Psf UF, without any notable variation in $\% \mathrm{SR}$. It is also observed through the obtained rejection behaviours that, the reinforcement of carboxylated CNTs remains adequately effective to maintain nearly similar solute rejections for the concerned mixed-matrix UF membranes, signifying the absence of noticeable imperfections in the polymeric host matrix even on the enhanced reinforcement of the nanoadditives.

The analyses of steady-state PWP of the UF membranes (Figure 9) illustrate a prominent change from 2000 LMD for the Control-Psf UF to $\sim 2300-2500$, $\sim 2400-2600$ and $\sim 2700-3000$ LMD for the mixedmatrix UF membranes reinforced with SWNT, DWNT and MWNT, respectively, on progressively higher concentration. The rise of PWP as happened in the mixed-matrix UF membranes on enhanced reinforcement of the pristine CNTs is predominantly attributed to the resulting membrane morphology that gets influenced by the phase inversion mechanism owing to the apparent hydrophobic nature of the pristine CNTs. The favoured secondary interaction between the pristine CNTs, at lesser concentration and polaraprotic solvent, NMP over that with polar-protic nonsolvent, water, as discussed earlier during analysis of hydrophilic features may retard the kinetics of both, non-solvent's indiffusion and the solvent's outdiffusion. Such phase inversion kinetics is known to impose comparatively more porous substructural features with dense skin layers in the respective membranous matrices [40, 41]. The moderate enhancement 
in PWP of the membranes as compared to the Control-Psf UF, with similar rejection behaviours corroborates the aforesaid features. However, the further increase of PWP with no such notable compromise in rejection capability, on reinforcement of the pristine CNTs at higher concentration is ascribed to the reciprocal contribution of the enhanced solution viscosity. This results in added delaying of the phase inversion leading to porous substructures and tentative irregularities owing to the inhomogeneous distribution of the aggregated CNTs within membranous matrices, probably creating several additional permeating routes [42]. Under this circumstance, the effect of decline in hydrophilicity and variation in $\zeta$ might have been overcompensated by the aforesaid physicochemical variations.

However, the facilitated reinforcement of the CSWNT, C-DWNT and C-MWNT within the membranous structures becomes noteworthy in amplifying the PWP of the respective mixed-matrix UF membranes. This gets observed through the variations represented in Figure 9, as $\sim 3500-3700$, $\sim 3600-4400$ and $\sim 4000-4500$ LMD, respectively, on enhanced reinforcement of the stimulated nanoadditives. The impact of functionalization pathway to enhance the solvent permeability of CNT reinforced mixed-matrix membranes has also been underpinned by other researches [11-13]. The carboxylated CNTs being enriched with hydrophilic functionalities expedite the kinetics of non-solvent's indiffusion and the solvent's outdiffusion, amending the phase inversion process through instantaneous demixing of the casting dope solutions. Such alteration in the phase inversion mechanism is familiar to result in formation of highly porous substructures and finely porous thin skin layers [40, 41]. The amplified permeabilities in conjunction with trivial variation in solute rejection capability, as obtained for the concerned mixed-matrix UF membranes corroborate the facilitated diffusive mass exchange between solvent and non-solvent, during the phase inversion process. Furthermore, the ultrafiltration performances substantiate the demonstrated facilitated distribution of carboxylated CNTs, even at higher concentration which impedes the notorious aggregation effect within the polymer matrices. The trends obtained for the ultrafiltration are in well accordance with the discussed variations in hydrophilic features. The high permeability of the membranes confers that the modified nanoadditives not only influence the physicochemical features of the membranes, but concomitantly infuse charge contribution in stimulating the membranes' porous pathways [43]. The molecular transport is predominantly controlled by the hydrodynamic interactions, but there may also exist the concurrent effect of electrostatic interactions for the mixed-matrix UF membranes. The implanted charged sites being part of the entrapped functionalized CNTs herein exert considerable electrostatic interactions with the diffusing solvent molecules, and thereby improve the hydrodynamic dragging efficiencies of the amendable porous pathways.

\section{Conclusions}

Mixed-matrix membranes were developed with impregnation of CNTs of different configurations, viz. SWNT, DWNT and MWNT, and their carboxylated counter-parts. The structural features, analyzed through XPS, revealed that MWNTs existing in clustering morphology with the most available surface area were favourably oxidized in comparison to its configurational counterparts. On the other hand, SWNTs, staying in bundles because of significant attractive forces remained incapable to offer enough reactive sites during oxidation. It was illustrated that the carboxylated CNTs, as superior nanoadditives compared to the pristine ones, led to the development of high-performance mixed-matrix membranes. The improved electrokinetic features indicated the role of the charged sites associated with the carboxylated CNTs in amending the surface conductivities of the membranes. The transport characteristics of the membranes confirmed the effect of reinforcement of carboxylated CNTs in contrast to the pristine ones at similar concentration, since the former was turned out to be more effective in increasing the solvent flux of the mixed-matrix UF membranes without compromise in their solute rejection capabilities. The alterations brought about in the phase inversion process of membrane making, fine-tuning of the membrane morphology, and the infusion of the charged sites over membrane surface were found responsible for the superior productivity of the carboxylated CNT reinforced mixed-matrix UF membranes. More importantly and precisely, the mixed-matrix UF membrane with $1\left(\mathrm{w} / \mathrm{wPsf}_{\mathrm{Ps}}\right) \%$ of carboxylated MWNT offered the best throughput amongst its configurational and carboxylated counter parts. 


\section{References}

[1] Qu X., Brame J., Li Q., Alvarez P. J. J.: Nanotechnology for a safe and sustainable water supply: Enabling integrated water treatment and reuse. Accounts of Chemical Research, 46, 834-843 (2013).

https://doi.org/10.1021/ar300029v

[2] Yin J., Deng B.: Polymer-matrix nanocomposite membranes for water treatment. Journal of Membrane Science, 479, 256-275 (2015).

https://doi.org/10.1016/j.memsci.2014.11.019

[3] Mittal G., Dhand V., Rhee K. Y., Park S-J., Lee W. R.: A review on carbon nanotubes and graphene as fillers in reinforced polymer nanocomposites. Journal of Industrial and Engineering Chemistry, 21, 11-25 (2015). https://doi.org/10.1016/j.jiec.2014.03.022

[4] Goh P. S., Ismail A. F., Ng B. C.: Carbon nanotubes for desalination: Performance evaluation and current hurdles. Desalination, 308, 2-14 (2013).

https://doi.org/10.1016/j.desal.2012.07.040

[5] Corry B.: Water and ion transport through functionalised carbon nanotubes: Implications for desalination technology. Energy \& Environmental Science, 4, 751-759 (2011). https://doi.org/10.1039/C0EE00481B

[6] Majumder M., Chopra N., Hinds B. J.: Effect of tip functionalization on transport through vertically oriented carbon nanotube membranes. Journal of the American Chemical Society, 127, 9062-9070 (2005).

https://doi.org/10.1021/ja043013b

[7] Misdan N., Ismail A., Hilal N.: Recent advances in the development of (bio)fouling resistant thin film composite membranes for desalination. Desalination, 380, 105 111 (2016).

https://doi.org/10.1016/j.desal.2015.06.001

[8] Kang S., Herzberg M., Rodrigues D. F., Elimelech M.: Antibacterial effects of carbon nanotubes: Size does matter! Langmuir, 24, 6409-6413 (2008).

https://doi.org/10.1021/la800951v

[9] Narayan R. J., Berry C., Brigmon R.: Structural and biological properties of carbon nanotube composite films. Materials Science and Engineering: B, 123, 123-129 (2005).

https://doi.org/10.1016/j.mseb.2005.07.007

[10] Kar S., Bindal R. C., Tewari P. K.: Carbon nanotube membranes for desalination and water purification: Challenges and opportunities. Nano Today, 7, 385-389 (2012). https://doi.org/10.1016/j.nantod.2012.09.002

[11] Yin J., Zhu G., Deng B.: Multi-walled carbon nanotubes (MWNTs)/polysulfone (PSU) mixed matrix hollow fiber membranes for enhanced water treatment. Journal of Membrane Science, 437, 237-248 (2013). https://doi.org/10.1016/j.memsci.2013.03.021
[12] Park C. H., Tocci E., Fontananova E., Bahattab M. A., Aljlil S. A., Drioli E.: Mixed matrix membranes containing functionalized multiwalled carbon nanotubes: Mesoscale simulation and experimental approach for optimizing dispersion. Journal of Membrane Science, 514, 195-209 (2016).

https://doi.org/10.1016/j.memsci.2016.04.011

[13] de Lannoy C-F., Soyer E., Wiesner M. R.: Optimizing carbon nanotube-reinforced polysulfone ultrafiltration membranes through carboxylic acid functionalization. Journal of Membrane Science, 447, 395-402 (2013). https://doi.org/10.1016/j.memsci.2013.07.023

[14] Ajayan P. M.: Nanotubes from carbon. Chemical Reviews, 99, 1787-1799 (1999). https://doi.org/10.1021/cr970102g

[15] Balasubramanian K., Burghard M.: Chemically functionalized carbon nanotubes. Small, 1, 180-192 (2005). https://doi.org/10.1002/smll.200400118

[16] Shen C., Brozena A. H., Wang Y.: Double-walled carbon nanotubes: Challenges and opportunities. Nanoscale, 3, 503-518 (2011). https://doi.org/10.1039/C0NR00620C

[17] Sahoo N. G., Rana S., Cho J. W., Li L., Chan S. H.: Polymer nanocomposites based on functionalized carbon nanotubes. Progress in Polymer Science, 35, 837-867 (2010).

https://doi.org/10.1016/j.progpolymsci.2010.03.002

[18] Yu H., Jin Y., Li Z., Peng F., Wang H.: Synthesis and characterization of sulfonated single-walled carbon nanotubes and their performance as solid acid catalyst. Journal of Solid State Chemistry, 181, 432-438 (2008). https://doi.org/10.1016/j.jssc.2007.12.017

[19] Tasis D., Tagmatarchis N., Bianco A., Prato M.: Chemistry of carbon nanotubes. Chemical Reviews, 106, 11051136 (2006). https://doi.org/10.1021/cr050569o

[20] Vaisman L., Wagner H. D., Marom G.: The role of surfactants in dispersion of carbon nanotubes. Advances in Colloid and Interface Science, 128-130, 37-46 (2006). https://doi.org/10.1016/j.cis.2006.11.007

[21] Alston J. R., Overson D., Poler J. C.: Direct measurement of the interactions of amide solvents with single-walled carbon nanotubes using isothermal titration calorimetry. Langmuir, 28, 264-271 (2012). https://doi.org/10.1021/la203765j

[22] Liu Y-L.: Effective approaches for the preparation of organo-modified multi-walled carbon nanotubes and the corresponding MWCNT/polymer nanocomposites. Polymer Journal, 48, 351-358 (2016).

https://doi.org/10.1038/pj.2015.132

[23] Andrews R., Weisenberger M. C.: Carbon nanotube polymer composites. Current Opinion in Solid State and Materials Science, 8, 31-37 (2004).

https://doi.org/10.1016/j.cossms.2003.10.006 
[24] Datsyuk V., Kalyva M., Papagelis K., Parthenios J., Tasis D., Siokou A., Kallitsis I., Galiotis C.: Chemical oxidation of multiwalled carbon nanotubes. Carbon, 46, 833-840 (2008).

https://doi.org/10.1016/j.carbon.2008.02.012

[25] Ago H., Kugler T., Cacialli F., Salaneck W., Shaffer M., Windle A., Friend R.: Work functions and surface functional groups of multiwall carbon nanotubes. The Journal of Physical Chemistry B, 103, 8116-8121 (1999). https://doi.org/10.1021/jp991659y

[26] Huang Y. Y., Terentjev E. M.: Dispersion of carbon nanotubes: Mixing, sonication, stabilization, and composite properties. Polymers, 4, 275-295 (2012).

https://doi.org/10.3390/polym4010275

[27] Zhbanov A. I., Pogorelov E. G., Chang Y-C.: Van der Waals interaction between two crossed carbon nanotubes. ACS Nano, 4, 5937-5945 (2010). https://doi.org/10.1021/nn100731u

[28] Brandão S. D. F., Andrada D., Mesquita A. F., Santos A. P., Gorgulho H. F., Paniago R., Pimenta M. A., Fantini C., Furtado C. A.: The influence of oxygen-containing functional groups on the dispersion of single-walled carbon nanotubes in amide solvents. Journal of Physics: Condensed Matter, 22, 334222/1-334222/8 (2010). https://doi.org/10.1088/0953-8984/22/33/334222

[29] Landi B. J., Ruf H. J., Worman J. J., Raffaelle R. P.: Effects of alkyl amide solvents on the dispersion of single-wall carbon nanotubes. The Journal of Physical Chemistry B, 108, 17089-17095 (2004).

https://doi.org/10.1021/jp047521j

[30] Coleman J. N.: Liquid-phase exfoliation of nanotubes and graphene. Advanced Functional Materials, 19, 3680 3695 (2009).

https://doi.org/10.1002/adfm.200901640

[31] O'Connell M. J., Eibergen E. E., Doorn S. K.: Chiral selectivity in the charge-transfer bleaching of single-walled carbon-nanotube spectra. Nature Materials, 4, 412-418 (2005). https://doi.org/10.1038/nmat1367

[32] Hasan T., Scardaci V., Tan P., Rozhin A. G., Milne W. I., Ferrari A. C.: Stabilization and 'debundling' of singlewall carbon nanotube dispersions in N-methyl-2-pyrrolidone (NMP) by polyvinylpyrrolidone (PVP). The Journal of Physical Chemistry C, 111, 12594-12602 (2007). https://doi.org/10.1021/jp0723012

[33] Ata S., Mizuno T., Nishizawa A., Subramaniam C., Futaba D. N., Hata K.: Influence of matching solubility parameter of polymer matrix and CNT on electrical conductivity of CNT/rubber composite. Scientific Reports, 4, 7232/1-7232/8 (2014).

https://doi.org/10.1038/srep07232

[34] Celik E., Park H., Choi H., Choi H.: Carbon nanotube blended polyethersulfone membranes for fouling control in water treatment. Water Research, 45, 274-282 (2011). https://doi.org/10.1016/j.watres.2010.07.060
[35] Ariza M. J., Benavente J.: Streaming potential along the surface of polysulfone membranes: A comparative study between two different experimental systems and determination of electrokinetic and adsorption parameters. Journal of Membrane Science, 190, 119-132 (2001). https://doi.org/10.1016/S0376-7388(01)00430-6

[36] Möckel D., Staude E., Dal-Cin M., Darcovich K., Guiver M.: Tangential flow streaming potential measurements: Hydrodynamic cell characterization and zeta potentials of carboxylated polysulfone membranes. Journal of Membrane Science, 145, 211-222 (1998). https://doi.org/10.1016/S0376-7388(98)00077-5

[37] Pal A., Dey T. K., Bindal R. C.: Intrinsic dependence of hydrophilic and electrokinetic features of positively charged thin film composite nanofiltration membranes on molecular weights of poly(ethyleneimine)s. Polymer, 93, 99-114 (2016). https://doi.org/10.1016/j.polymer.2016.04.026

[38] Ma P-C., Siddiqui N. A., Marom G., Kim J-K.: Dispersion and functionalization of carbon nanotubes for polymer-based nanocomposites: A review. Composites Part A: Applied Science and Manufacturing, 41, 13451367 (2010).

https://doi.org/10.1016/j.compositesa.2010.07.003

[39] Chowdhury S. C., (Gama) Haque B. Z., Okabe T., Gillespie Jr. J. W.: Modeling the effect of statistical variations in length and diameter of randomly oriented CNTs on the properties of CNT reinforced nanocomposites. Composites Part B: Engineering, 43, 17561762 (2012).

https://doi.org/10.1016/j.compositesb.2012.01.066

[40] Yun Y., Le-Clech P., Dong G., Sun D., Wang Y., Qin P., Chen Z., Li J., Chen C.: Formation kinetics and characterization of polyphthalazinone ether ketone hollow fiber ultrafiltration membranes. Journal of Membrane Science, 389, 416-423 (2012).

https://doi.org/10.1016/j.memsci.2011.11.007

[41] Wang D-M., Lin F-C., Chiang J-C., Lai J-Y.: Control of the porosity of asymmetric TPX membranes. Journal of Membrane Science, 141, 1-12 (1998).

https://doi.org/10.1016/S0376-7388(97)00273-1

[42] Qui S., Wu L., Pan X., Zhang L., Chen H., Gao C.: Preparation and properties of functionalized carbon nanotube/PSF blend ultrafiltration membranes. Journal of Membrane Science, 342, 165-172 (2009). https://doi.org/10.1016/j.memsci.2009.06.041

[43] Kar S., Pal A., Subramanian M., Nuwad J., Bindal R. C., Chattopadhyay S., Tewari P. K.: High-throughput mixed-matrix membrane with superior anti-bacterial properties: A facile approach towards development of point-of-use water purification device. Chemical Engineering Journal, 297, 193-206 (2016). https://doi.org/10.1016/j.cej.2016.03.147 